\title{
The Cultural Dimension and Its Influence on the Relationship between the Textile and Furniture
}

\author{
Dr. Inas Hosny Ibrahim Anous \\ Lecturer ,Faculty of Applied Arts, Depatement of Interior Design and \\ Helwan University- Egypt
}

Doi:10.5901/mjss.2013.v4n11p295

\section{Abstract}

The tapestry contribute to the charm of interior design and comfort of men, its delicate colours complete the style of furniture , certain tapestries, in robust drawing, combine very well with a rustique or contemporary furniture. The tapestries of chairs, "au petit point", naturally accompany the old wood as those of the Beauvais factory. With branches and flowers under the reign of Louis XIV, with medallions under the reign of Louis XVI, with ancient scenes in the Empire, in round bouquets under the reign of Louis-Philippe, the tapestry seat have their frames or their background animated by the decorative themes which characterize each style. The research aims to examine the close relationship between the textile and furniture in the light of The cultural dimension of each society from the XVII century to the XX Century in France.

Keywords: fabric styles - tapestry- furniture style

\section{Introduction}

Le siège, très souvent objet des arts décoratifs, est parfois aussi un modèle ou une oeuvre artistique accomplie en ellemême. Depuis l'origine, les tissus jouent un grand rôle dans l'art du mobilier, notamment pour les garnitures de sièges. L'étude du textile des sièges passe obligatoirement par la connaissance des différents styles. Les tissus se distinguent selon les matières premières dont ils sont composés, selon la manière dont ils sont tissés et selon la façon dont le décor est obtenu. D'un point de vue stylistique, l'éventail des tendances est large d'un siècle à l'autre : du néoclassicisme des sièges Louis XVI, on passe, quelques années après la Révolution, aux formes néopompéiénnes et dites " à l'étrusque " sous le Directoire. Les lignes s'affinent sous Napoléon 1er avant de rebasculer vers le goût des étoffes drapées et les garnitures complexes sous le Second Empire. L'Art nouveau sera économe en garnitures et utilisera plutôt des bois blancs et des étoffes d'une grande créativité graphique.

\section{Problème de la recherche}

1. Peu de references qui traitent le textile et l'art du tapissier à travers les époques, par opposition à l'art des ébénistes ou des menuisiers.

2. Peu de sièges de styles ont pu être maintenues sur leurs supports primitives, dont l'exactitude serait difficile à prouver, ce qui forme un vrai problem de référence devant l'architecte d'intérieure en cas ou il a besoin de créer l'atmosphère d'un certain style de décor à un espace intérieure.

\section{L'objectif de la recherche}

1. Etude de l'influence de la dimension culturelle sur la relation entre le textile et le mobilier de style.

2. Analyse alternée des étoffes et du mobilier correspondant aux différentes époques ce qui permet de composer un ensemble "d'époque" sans erreur.

\section{Méthodologie}

La recherche se base sur l'étude du textile; genres, ornements et couleurs concernant les styles de sièges français à travers les époques de l'histoire allant du XVII siècle jusqu'au XX siècle. La recherche commence par l'étude des dimensions culturelles qui influencent la relation entre le textile et le siège dans les différents styles ainsi que celles 
présents durant les périodes de transitions. Au deuxième rang, vient l'aspect du siège ; ligne, forme et bois. Troisièment, la recherche va preciser quel textile pour quel style de siège dans les ensembles décoratifs. Le premier style à mettre en valeur est le style "Louis XIII qui est suivi par le style "Louis XIV". Passant par la Régence, elle aboutit au Style "Louis XV". La Transition précède le style "Louis XVI". Le style Directoire conduit au style Empire. Le style Restauration, suivi par le style Louis- Philippe et le second Empire qui termine cette phase. Au XX siècle, la recherche met en valeur le style Art-Nouveau et l'Art déco.

\section{Analyses}

\subsection{Style Louis XIII (1589-1661)}

\subsubsection{La dimension culturelle influant les sièges}

Bienque la France est devenu la plus grande nation d'Europe, ce n'est elle qui donne le ton.On voit s'y entrecroiser des influences espagnoles, italiennes, flamandes, qui prennent l'aspect de véritables modes. Après les guerres de Religion et le climat d'insécurité ,les français éprouvent un certain besoin de confort et de stabilité. C'est une époque de transformation pour Paris, qui s'embellit de maisons particulières et d'édifices publics. La France résiste aux styles baroques qui vont s'étendre en Europe. l'Espagne est le pays le plus puissant économiquement et militairement en Europe et son influence s'étendait jusqu'au sièges (Feray1991).

\subsubsection{Les sièges:}

Caractéristiques des sièges et bois : Avec la montée de la classe moyenne, la vie familiale se développe. La chaise à bras- fauteuil- devient une "commodité pour la conversation". Grâce à la sobriété de sa forme, elle devient plus confortable et peut facilement être déplacée. L'assise s'abaisse et se garnie d'un rembourage. On la recouvre avec des tissus, de la tapisserie aux riches décors ou avec du cuir repoussé. Les chaises reprenaient la forme trapue hispanoflamande, un peu raide avec un siège bas et un dossier peu élevé.Le siège Louis XIII est caractérisé par des lignes droites, son aspect sévère, tempéré par la richesse de la décoration. Les pieds sont parfois en forme de colonne, s'élevant d'un châssis carré à boules, mais la plupart présentent un piètement en balustres ou à décor tourné, relié par une entretoise en forme de $\mathrm{H}$, avec les pieds antérieurs joints, dans la partie supérieure, par une traverse de renfort supplémentaire d'aspect décoratif. Le siège canné, d'origine hollandaise, est exceptionnel à cette époque. II allie le tournage à la sculpture. A la fin du règne Louis XIII, le tournage- sous l'influence espagnole- devient plus complexe.Les bois utilisés sont: le hêtre et le noyer ( Castellucio2002).

\subsubsection{Les tissus:}

- Genres des tissus: Les étoffes provenaient d'Espagne et d'Italie en particulier les velours: de lin , de cotton ou de soie uni, velours de Gênes ou brodés ou façonnés, soies (taffetas, satin...) et damas de coton, tavaïolle, serge, toile brochée, dentelle, Catalogne (drap fin), toile d'argent. Ces étoffes sont lourdes et presque toujours brodées ou soulignées de passementeries et de franges. La broderie, de fils de soie, couvraient entièrement les sièges et permettaient la confection des décors complexes, décoratifs ou historiés .L'emploi de l'or et de l'argent ajoutaient à la l'effet de la couleur celui de la richesse. Les soieries tiennent une place importante dans la couverture des sièges. Les cuirs sont un moyen de couverture très en vogue parmi lesquels; le cuir de Cordoue relevé d'or. Les tapisseries moins complexes; les materiaux employés sont le lin pour le canevas, la laine et la soie pour les motifs et réalisés au point de croix.Les tapisseries aux petits points avec des motifs très colorés comme "le fameux point de Hongrie" qui se compose de chevrons emboités, qui affirmait sa maitrise de couleurs de fils de laine et de soie. La tapisserie couvrait entièrement dossier et le siège des fauteuils.Sur le siège Fig( 2), un rectangle de broderie de soie blanche appliqué occupe le centre du dossier et du siege, entouré de quatre panneaux de velours de genes cramoisi, dont les coutures en diagonals et le tour sont soulignes d'une bordure de soie blanche (Morand. 1973 ).

- Ornements des tissus: les motifs Mille fleurs sont très appréciés. emblèmes héraldiques,Beaucoup de feuilles d'acanthe, de fleurs, de ramages, de raies larges, petits carreaux, des scènes champêtres, bibliques, historiques ou mythologiques, sujets de chasse, bêtes, ciseaux, fleurs, ramages et verdures 
decorent les tapisseries. (Ossut 2002)

- Couleurs des tissus: Le rouge, le vert et le jaune apparaissent comme les teintes les plus habituelles. Fig( 1 )
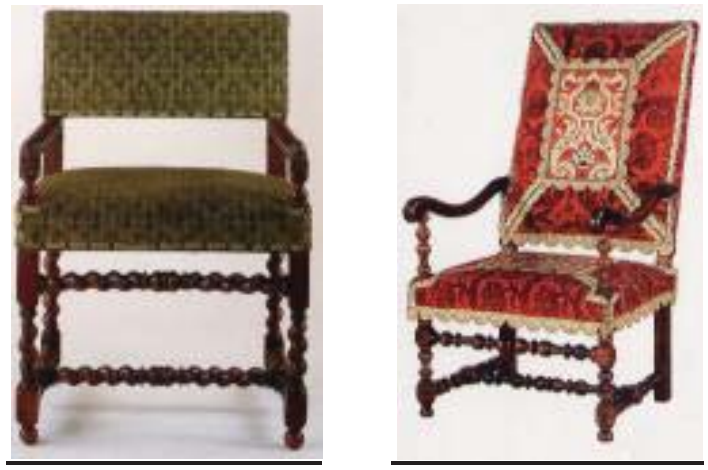

Fig. 1 siège et tissue style Louis XIII

Fig .2 siège et tissu style Louis XIII

Source: Castellucio, S. (2002)p48.49.

\subsection{Style Louis XIV (1661-1715)}

\subsubsection{La dimension culturelle influant les sièges}

Les différences entre les classes sociales étaient bien marquées, ces inégalités se manifestaient dans tous les domaines de la vie.En 1684, et même à l'apogée du règne de Louis XIV, la masse du peuple demeurant aussi ignorante qu'au moyen-âge ; les fils des nobles et des riches bourgeois étaient instruits. L'éclat du Roi- soleil civilise la noblesse dont elle forme le goût.A Paris, des centaines des nobles et des riches bourgeois vivaient dans des demeures de luxe : les hôtels particuliers bienque la majorité du peuple vivait dans la misère.

Le mobilier Louis XIV est le plus royal de tous les styles francais.Tous les meubles crées pour les appartements du roi à Verailles - avec ses jardins "à la française" - sont des prototypes.A la cour comme dans les chàteaux, le choix des sièges suit une étiquette et un ordre hiérarchique. Aux princes les fauteuils, aux seigneurs les plus titrés les chaises, aux courtisans les tabourets, aux "dames d'atours" les coussins posés sur le sol..( Feray1997)

\subsubsection{Les sièges:}

Caractéristiques des sièges et bois : La structure du grand fauteuil reste celle du règne précédent.Elle est dominée par la rigeur. Aucun type de meuble ne personnifie mieux les caractéristiques du Louis XIV et de la période que le sévère fauteuil rembourré, imposant dans son immobile force. Plusieurs éléments contribuent à l'impression de grandeur qui s'en dégage, notamment son dossier rectangulaire, légèrement incliné, entièrement recouvert d'étoffe et excessivement haut, sa large assise, ses accotoirs se terminant par une volute et ses pieds solidement liés par une lourde entretoise en $\mathrm{H}$ ou en $\mathrm{X}$. Le piètement est tour à tour constitué de balustres en bois tourné, d'éléments en forme de gaine ou d'enroulements. En avancant dans le siècle, les bois s'enrichissent de sculptures alternant avec des parties tournées; les pieds accusent un galbe pronouncé et ornés de fins motifs sculptés Fig( 3 ). Les sièges sont tournés ou sculptés en bois de hêtre ou en noyer.

\subsubsection{Les tissus:}

- Genres des tissus: Les tissus sont d'une variété extrême et d'une polychromie éclatante . A l'image de la grandeur du roi, les étoffes précieuses sont largement utilisées. Les soieries à motifs alors en usage sont rehaussées de fils d'or ou d'argent. Leur décoration est le plus souvent symétrique.On trouve les velours et les satins brodés d'or et d'argent ; les soies unies, les taffetas jaunes, la moire, les soies façonnées, le satin blanc, les damas rouge cramoisi, aurore ou les damas bleus à bandes de tapisserie, les velours de toutes 
couleurs, le velours d'Utrecht (velours en laine mohair sur fond de lin de couleurs opposés) ainsi que le gros de Tours. les brocarts, les draps d'or et d'argent, le velours uni : peluche de Chine, les tissus de Perse, la toile des Indes, la gaze rayée, la serge verte, l'étoffe à petits carreaux, les dentelles, la toile fine brodée, parfois les mousselines. Tapisserie au petit point, au point de Hongrie. Tissées d'Aubusson, d'Auvergne, des Gobelins, d'Angleterre et de Beauvais. Toutes les garnitures sont, en général, bordées de galon d'or, de frange de soie, ou de laine, autour de la ceinture (Demetrescu 2002).

- Ornements des tissus Aux jardins "à la française" du 17eme siècle correspond la rigeur de la composition florales des tissus. Gros fruits entourés de branchages, grands motifs en ananas, grosses fleurs, feuillages et feuilles d'acanthe, arabesques, bouquets, ifs, caisses d'oranger, coquillages, dieux, paysages, arabesques ; pour les tapisseries : exploits guerriers, graves événements, sujets empruntés à l'antiquité et à l'Ancien Testament, saisons, chasses de Bruxelles.

- Couleurs des tissus: Les couleurs restent vivent et la couleur rouge, symbole de pouvoir y est très présente ainsi que le vert, le bleu et le jaune Fig(4).
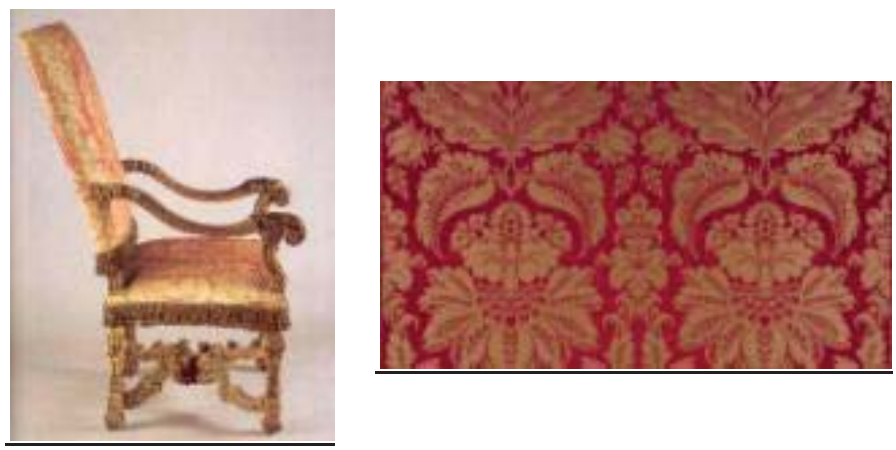

Fig. 3 siège et tissu Louis style XIV

Fig. 4 Damas rouge style Louis XIV

Source: http://tldtouteladecoration.com/etoffes-du-xviieme-louis-xiv(accès 27/6/2013)

\subsection{Le style Régence (1715-1730)}

\subsubsection{La dimension culturelle influant les sièges:}

L'étiquette rigide du règne de Louis XIV séloigne. Le rétablissement des relations avec la Grande Bretagne ramène la vogue du cannage des sièges, apprécié pour sa légèreté et sa résistance.La cour est désormais à Paris et non plus dans les antichambres de Versailles; elle ne tient plus debout devant le roi mais cherche ses aises en s'asseyant dans les salons. La régence est synonyme de la vivacité intellectuelle ,légèreté pleine d'équilibre et de délicatesse.Les artistes et les artisans ne travaillent plus guère pour le monarque et sa cour; les commandes leur viennent des particuliers qui s'échappent à la containte asphyxiante de l'époque précédente( Demetrescu 2003).

\subsubsection{Les sièges:}

Caractéristiques des sièges et bois : L'étude des chaises donne la vision la plus complète de l'évolution ou de la transition entre le Louis XIV et le Louis XV. Le style Régence conserve les lignes architecturales du style Louis XIV. Les formes s'assouplissent, les pieds se galbent Fig(5). Pour faire place aux robes à paniers, les accotoirs se mettent en retrait, une traverse frontale en bois sculpté formant avec le piètement une ligne sinueuse ininterrompue, puis la disparition progressive de l'entretoise et finalement le choix d'un dossier encadré d'un châssis en bois apparent. Les éléments Louis XIV sont les montants rectilignes du dossier et la forme quadrangulaire de l'assise. La transition, parfaitement accomplie, la chaise ne possédera plus une seule ligne droite, annonçant ainsi le style Louis XV.La garniture cède la place au bois apparent : doré pour les sièges d'apparat, vernis ou au naturel . Le hêtre, le noyer, les bois fruitiers et le tilleul servent à la fabrication des sièges. (Bedel 2004) 


\subsubsection{Les tissus:}

- Genres des tissus: Les sièges sont recouverts de tapisserie, de velours ou de soie. Proches de ceux du style Louis XIV : tapisseries de Beauvais, Aubusson et Gobelins. Soies façonnées, soieries à bouquets, des lampas, des brocatelles, damas, satin, brocart jaune or et gris, velours et satins brochés verts, rouges et blancs, velours et soies unis. Les étoffes sont souvent garnies de galons très ornés( Demetrescu 2003)

- Ornements des tissus: Souvent d'inspiration florale, grosses fleurs et gros fruits, reproduction de dentelles sur fond de couleur, semis en quinconce. Les motifs sont de moins en moins symétriques.

- Couleurs des tissus:: Le bleu dur, le rouge, le vert, le jaune, le blanc, le gris et l'or.

Fig. 5 siège et tissu Regence

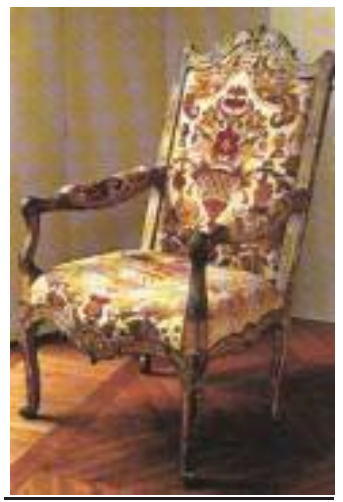

Source: Demetrescu ,C.(2003)p.66

\subsection{Style Louis XV (1730-1760)}

\subsubsection{La dimension culturelle influant les sièges:}

Un vent de frivolité à balayer Lère du protocole des la période de la Régence.Le style Louis XV est créer dans un esprit de confort et de plaisir sans précédant par une société galante très influencée par le baroque italien et les arts orientaux (Indes, Chine, Turquie) ;Il règne aussi dans l'air un désir d'exotisme et les étoffes sont décorées de Chinoiserie, d'oiseux merveilleux de Singeries.. La vie à la cours restera faste jusqu'en 1760 et L'engouement pour «'art de vivre » progressant les demeures deviennent plus intimes et plus confortable. Le mobilier Louis XV est donc charmant, élégant, léger. Les artistes se revendiquent détachés des anciens codes, de la rigidité, de la symétrie et de l'austérité des anciens styles et L'ornementation devient rocaille, courbe et contre courbe. II est aussi symbole de création et de féminisme. En effet les commandes sont essentiellement faite par ou pour les femmes qui, prenant une place des plus importante à la cour décidèrent aussi des tendances en matière de décoration. Les deux femmes emblématiques en sont la Marquise de Pompadour et la Comtesse de Barry. A la même époque, un nombre de plus en plus grand de bois indigènes et exotiques inondaient le marché.Trois raisons expliquent la mode des indiennes: La Compagnie française des Indes orientales, Les relations entre la France et l'Orient se développent et Ces étoffes sont légères, colorées et leurs couleurs résistent aux lavages.C'est une époque de tranquillité interieure, richesse en hommes, organisation de la société et des métiers d'art( Mouquin 2003).

\subsubsection{Les sièges:}

Caractéristiques des sièges et bois : Le fauteuil cabriolet apparaît vers 1740. C'est le premier fauteuil dont le dossier s'incurve pour épouser la forme du dos à la façon du siège de la voiture légère à laquelle il doit son nom. II est confortable et élégant. Les éléments qui le composent paraissent se couler les uns dans les autres, sans la moindre interruption de la ligne sinueuse qui devient englobante. Les châssis des chaises sont agrémentés de riches sculptures 
de fleurettes, feuillages et coquilles. Le type de siège le plus caractéristique du Louis XV est la bergère, un large et profond fauteuil à dossier enveloppant dit en gondole. Les canapés se déclinent en une grande variété : les sofas(à siège surbaisse) ; l'ottomane (à dossier en corbeille) ; la veilleuse (à accotoirs inegaux)...Bienqu'une grande variété de bois soit employée sous Louis XV , les sièges sont le plus souvent en hêtre ou quelque fois en noyer.La plupart des sièges sont peints Fig 6, notamment les sièges qui s'harmonisent avec les boiseries et le décor mural, d'où l'inutilité de l'emploi d'essences de bois au veinage particulier. Ils sont rehaussés d'un rechampi sur la mouluration.Parfois les sculptures sont dorées à la feuille comme dans le cas des sièges royaux (Jarry, Devinoy1973).

\subsubsection{Les tissus:}

- Genres des tissus: Le style Louis XV est précieux ; il réclame des tissus raffinés, élégants, luxueux.Les soieries sont a l'honneur, les indiennes qui sont des toiles imprimées de Perse ou des Indes. Toile de Jouy, Velours, lampas, damas, shantung, brocard, chintz glacé , taffetas, moire, satin . La tapisserie est moins à la mode : tapisserie au petit point qui representent pastorales, chinoiseries et fleurs, elle dédaigne les sujets solennels du Louis XIV . Les tissus de cette période présentent des effets de dentelle, des grosses fleurs, une nature luxuriante. La symétrie et les lignes droites laissent place aux formes chantournées et à la courbe. C'est l'apogée des soieries bizarres, ainsi nommées en raison de leurs motifs étranges et asymétriques.Parfois. une garniture de cuir ou de canne pour les fauteuils de bureau( Wiuegandt 1994).

Fig. 6 Fauteuil à la reine à chassis au bois peint style Louis XV.

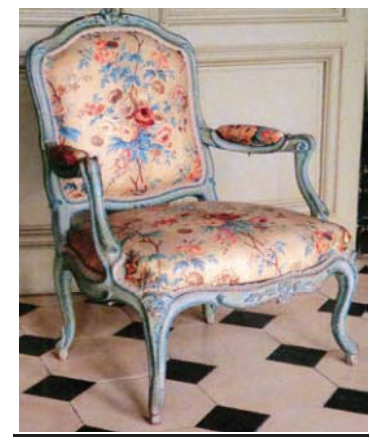

Source: Ossut,C. (2002)p.32

- Ornements des tissus :La dimension des motifs est rétrécis par rapport au style précédant et deviennent plus fins: fleurs, feuillages, branchages entremêlés, rubans, chinoiseries pour les soieries, des scènes campagnardes pour la toile de jouy. oiseaux, animaux, bouquets, rubans, branches fleuries, nœuds, grandes lignes sinueuses de tiges et de guirlandes réunissant bouquets et branches. Pour les tapisseries : attributs champêtres, chinoiseries très à la mode, pierrots, arlequins, colombines, fables de La Fontaine. Les tissus de cette période présentent de grosses fleurs, des formes asymétriques, des scènes exotiques ou des effets de dentelles en bandes ondulantes mêlés à des fleurs. Les bandes de fourrures deviennent un élément récurrent dans les tissus.

- Couleurs des tissus: les étoffes prennent des tons frais et souvent tendres, des reflets glacés, Les couleurs vives cohabitent avec des tons pastels, le jaune, le rose et le bleu lapis lazuli sont très en vogue mais aussi abricot, vert pâle, vieux rouge, cramoisi, blanc se marient aux couleurs de base( Ossut 2002).

\subsection{Style Louis XVI (1760-1789)}

\subsubsection{La dimension culturelle influant les sièges:}

Le style Louis XVI témoigne d'une période charnière de l'histoire marquée par la découverte d'Herculanum(1738) et 
Pompéi (1748) ; un véritable engouement néoclassique qui influença les arts décoratifs. La tendance néo-classique, née sous Louis XV, prend toute son ampleur dans le style Louis XVI.Mme de Pompadour, déléguée par Louis XV auX affaires culturelles, commande des meubles «à la greque ». L'antique chasse la rocaille.Le «Recueil d'antiquités égyptiennes, étrusques, greques, romaines et gauloise » de Caylus remet à l'honneur les motifs puisés dans le monde gréco-romain ( Droguet 2004).C'est Marie Antoinette qui donne le ton.

\subsubsection{Les sièges:}

Caractéristiques des sièges et bois : Le galbe disparait, la ligne droite revient en force. Si la rigueur prédomine la forme, les ornements sculptés d'une très grande variété viennent l'enrichir. En 1776 est créé, pour le comte d'Artois, un nouveau fauteuil au siège circulaire et au dossier en médaillon oblong. Cette forme de dossier va connaître une grande vogue tant pour les sièges à la Reine que le cabriolet. Sous Louis XVI,la création des menuisiers s'est exprimée avec plus de fantaisie: grande variété dans la forme des dossiers.Le hêtre facile à peindre ou le noyer qui se prête au sculpture.les sièges dorés sont réservés pour le mobilier d'apparat (Kjellberg1991).

\subsubsection{Les tissus:}

- Genres des tissus: Les soieries de Lyon côtoient des perses et des tissus imprimés. La toile de Jouy s'imposera de plus en plus en raison de son caractère économique.Les tissus les plus courants sont : damas, lampas, satin, aux coloris tendres, imberlines, moires et velours, voiles des Indes, le gourgouran apparaît. Tapisserie d'Aubusson et des Gobelins, surtout employées pour les fauteuils et les canapés : pastorales, jeux d'enfants, chinoiseries, bouquets entourés de rubans ou décorée de scènes champêtres, temples, statues antiques Les bordures sont plus simples et plus pauvres : fleurs, amours, guirlandes.

- Ornements des tissus: Les tapisseries se couvrent d'une multitude de fleurettes, de scènes champêtres, d'oiseaux...Les dessins s'affinent et sont d'une variété extraordinaire. Un créateur célèbre en soierie est: Philippe de la Salle. attributs pastoraux, scènes d'éducation maternelle, pipeaux, sujets mythologiques, instruments de musique, oiseaux, fleurs, scènes champêtres, plaisirs de la ferme, loups, moutons, chasse au cerf, centaures, décors orientaux, damiers, carreaux, motifs chinois, rubans, nœuds et rayures, médaillons, serpentins, semis de fleurs, plumes. Les satins ont des rayures coupées de fleurs, les damas sont décorés de bouquets de rosés. Les tissus de cette période présentent des rayures et paniers fleuris, des effets de fourrures, des passementeries dans une composition symétrique( Morand 1973).

- Couleurs des tissus: pastel et douces, souvent bleu ciel et rose Fig 7.

Fig. 7 Fauteuil au bois doré style Louis XVI livré par Georges Jacob pour le Grand Cabinet de la reine à Versailles en 1783

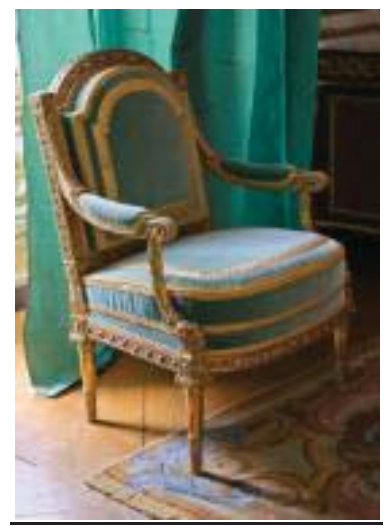

Source:http://fr.wikipedia.org/wiki/Style_Louis_XVI(accès25/5/2013) 


\subsection{Style Empire (1804-1815)}

\subsubsection{La dimension culturelle influant les sièges:}

Le style Empire recouvre la période de règne de Napoléon ler .L'anticomanie envahissant déjà les arts décoratifs sous Louis XVI et le Directoire et maintenant à son apogée. Ce style ne marque donc pas de rupture mais une continuité avec les styles précédents. Avec le retour de l'expédition d'Egypte, les décors égyptiens se développent.Le début du XIXe siècle sera marqué par l'époque napoléonienne et l'Empereur a voulu imposer au monde artistique l'expression de sa grandeur. Pour cela il va établir une centralisation rigide de toutes les activités artistiques qui seront désormais centralisées par Paris et dirigées par les architectes Percier et Fontaine et le peintre David. Les anciennes académies sont supprimées et remplacées par des expositions où des récompenses sont distribuées aux œuvres qui respectent le style imposé (Bedel 2004)

\subsubsection{Les sièges:}

Caractéristiques des sièges et bois : les décors égyptiens, ornementent des sièges au dossier rectangulaire, aux lignes géométriques, à la silouhette équilibrée et solennelle. Des bronzes ciselés et dorés leur donnent de l'éclat L'acajou triomphe jusqu'en 1806, date du blocus décrété par Napoléon 1er, qui ferme le continent au commerce britannique. Cessant d'arriver en France, il est remplacé par des bois indigènes, parfois teintés "façon acajou".

\subsubsection{Les tissus:}

- Genres des tissus: La toile de Jouy s'imposera de plus en plus en raison de son caractère économique. La toile de jouy represente Paul et Virginie, des fables de la Fontaine ou des scenes mytologiques, crin uni en losanges. Le velours uni ou frappé, très prisé, s'accompagne de soie, de satin, de taffetas. Le tissage de la soie atteint une perfection sans égal et Les étoffes sont le lampas, la brocatelle, le damas, les gros de Tour, les brochés et les brocards. En raison de leur solidité le tissu de crin, les tapisseries et les velours (qui peuvent être ciselés ou gaufrés) sont également très appréciés par l'empereur ( Morand1973). Complément indispensable du tissu : le galon de style généralement bicolore. Uni ou à motifs le tissu est toujours souligné de galon.

- Ornements des tissus: les étoffes étant le reflet du pouvoir impérial les motifs en sont marqués : le $\mathrm{N}$; l'abeille, le flambeau, la rosace, la palmette, les motifs sont généralement géométriques et les instruments de musiques toujours représentés.Semis en losanges, couronnes de laurier,feuilles de chêne, étoiles, palmettes, abeilles, marguerites, médaillons avec motifs de cygnes Fig8, rayures, faisceaux, motifs égyptiens, motifs antiques, motifs étrusques( Jarry, Devinoy 1973).

- Couleurs des tissus : vives ; La couleur de l'époque est bien sûr le vert empire, un vert profond, mais aussi le carmin, le bleu nuit et le beige parfois agrémenté de motifs jaune d'or. vives ; rouge, vert, jaune, apparition du violet .

Fig. 8 Fauteuil style Empire

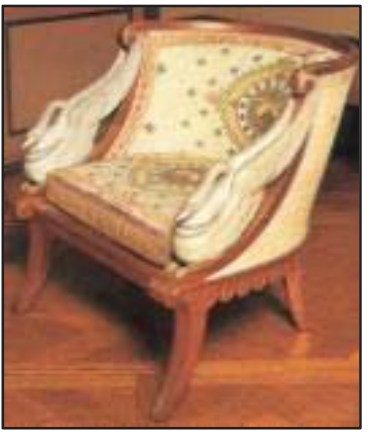

Source: Jarry .M , Devinoy. P.( 1973)p.206 


\subsection{Restauration (1815-1830)}

\subsubsection{La dimension culturelle influant les sièges:}

La Restauration est une réaction contre les fastes voyants et la pompe monumentale de l'Empire Il consiste en un retour à la souveraineté monarchique et voit donc l'expérience d'une monarchie constitutionnelle, avec une période relative de paix dans un contexte international troublé. La Révolution industrielle provoque des bouleversements économiques et sociaux profonds. Les dépenses sont aussi faibles que les finances avec peu de commandes d'apparat .Seule la duchesse de Berry introduit un peu de vie et de gaieté dans la cour. La duchesse de Berry, Marie Caroline de Naples, Marie Caroline de Naples figures emblématiques de la coure influenceront les arts décoratifs: personnalités très dépensières, elles commandent de nombreux meubles et font construire des bâtiments. On poursuit la tradition néoclassique de l'époque precedente. Le romantisme à la mode est loin de l'ambition brutale de l'empereur vaincu(Feray 1997). .Les jeunes qui encouragent le romantisme, cherchent leur inspiration dans un moyen âge imaginaire qui mêle l'ornementation classique du décor aux ogives, flèches et rosaces gothiques.

\subsubsection{Les sièges:}

Caractéristiques des sièges et bois :Les formes s'assouplissent, la marqueterie revient. Le fauteuil en gondole connaît un grand succès. De petite dimension, simple, léger et élégant, il restera en vogue jusqu'en 1840.Les sièges se distinguent par des pieds arrière en sabre et des pieds avant en fuseau, en balustre, en console.L'inspiration gothique fait naître des dossiers ajourés en ogive, les pieds avant galbés, les pieds arrière en sabre. En opposition aux sombres acajous, la faveur est aux bois clairs enrichis de volutes d'amarante, de palissandre le jaune pour les bois foncés Fig 9 .

\subsubsection{Les tissus:}

- Genres des tissus: Les étoffes utilisées restent les mêmes que sous l'empire.Toile de jouy avec des scènes de chasse à courre, crin a palmettes ou à mosaiques, soie, shantung, lumetis,velours, satin, percale glacée .

- Ornements des tissus: Avec le retour des Bourbons sur le trône de France, les fleurs de lys se substituent aux aigles et autres emblèmes de l'Empire. Des dessins répétitifs de petites dimensions. Les motifs centraux sont toujours en usage : des motifs rond très décorés suggérant une couronne ou une riche rosace sur un fond unis ou en répétition. On les distingue parfois des tissus Empire par la présence d'attributs royaux comme le lys ou le dauphin.Bouquets de fleurs ronds, feuillages, arabesques, scènes mytologiques, guirlandes de fleurs. L'égyptomanie passe un peu de mode mais les palmettes et les rinceaux sont massivement utilisés, accompagnés de rosaces, cygnes, volutes graphiques et feuillages (Kjellberg1991). Le style troubadour des années 1820-1930 remet à l'honneur les ornements gothiques après adaptation : ogives, rosaces, fleurons, guirlande de lierre et de vigne, fenestrages.

- Couleurs : jaunes vifs, tous les verts francs. Les tons acides : bleu opalin, vert mousse, rose, le turquoise.

Fig. 9 Fauteuil et tissu style Restauration

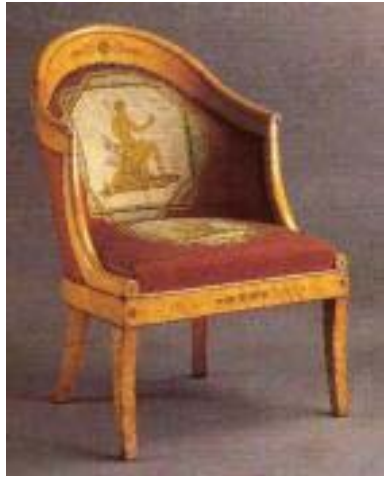

Source: http://www.confort-decor.ch(acces 26/5/2013) 


\subsection{Le Style Louis-Philippe (1830-1850)}

\subsubsection{La dimension culturelle influant les sièges:}

Louis Philippe est le dernier roi de France qui donnera son nom à un style. Après l'échec de la révolution romantique , la bourgeoisie régnant s'identifie volontiers à son souverain. Le roi lui- même n'a aucune influence sur le style. Ayant vecu en Angleterre, il se veut moderne et se contente d'un confort rembourré, dans une prospérité économique. Ce style bourgeois allie entre le desir du confort, la mécanisation du travail et la fabrication en série d'après les prototypes anciens.

\subsubsection{Les sièges:}

Caractéristiques des sièges et bois : Les pieds des sièges sont tournés à la machine. Les ébénistes et menuisiers offrent des sièges bien montés et solides.L'héritage de la Restauration accentue l'évolution des courbes; fauteuils à courbure accentuée et accotoires en volutes. Le fauteuil Voltaire voit son dossier se rembourrer, s'arrondir.Les bergères bien joufflués sont souvent "en gondole" et comportment des appuis-tete latéraux(Feray 1997). Les bois sombres se substituent aux bois clairs de la période précédente : acajou, palissandre, ebene, bois noircis(hetre et Poirier).Les bois traditionnels sont employes: noyer, érable, merisier. Les bois sont polis ou vernis à chaud, ce qui leur donne plus d'éclat(Morand1973).

\subsubsection{Les tissus:}

- Genres des tissus: Le velours connaît un grand succès sous le règne de Louis- Philippe.Toile de Jouy avec des scènes de chasse à courre, percale, chintz, feutre, shantung, satin, mat, percale glacée, crin, velours, satin fermière( Ossut 2002).

- Ornements des tissus: fleurs mêlées, gros bouquets, motifs orientaux, motifs perses, rayures larges, alternance des raies et de fleurs, motifs geometriques, pois.Volutes, crosses, palmettes et feuilles à larges petals.Le motif en "cuisse de grenouille" stylisé et en leger relief est très caractéristique Fig 10 .

- Couleurs des tissus : Le rouge, le grenat et le vert, le noir contenant du rouge et quelques camaieux.

Fig. 10 Fauteuil en acajou style Louis Philippe

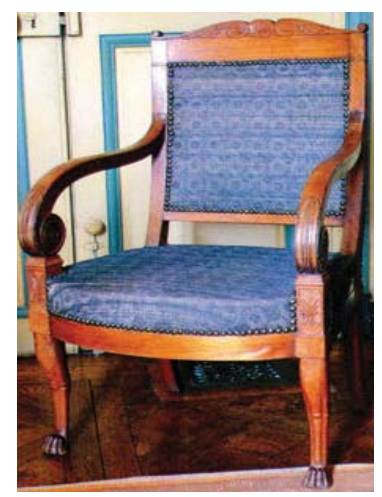

Source: Ossut,C. (2002)p.70

\subsection{Napoléon III (second Empire )(1850-1890)}

\subsubsection{La dimension culturelle influant les sièges:}

C'est un règne de faste et de consommation (on dépense enfin après avoir économisé sous Louis-Philippes), mais c'est dominée par la femme (et non l' homme et l'architecte). L'impératrice Eugénie, fervente admiratrice de Marie- 
Antoinette, régnera presque autant que son mari et inclinait vers un retour aux styles monarchiques. Paris connaît l'essor de nouvelles courtisanes devenues personnages publiques : La Païva, Cora, Pearl.Une nouvelle classe bourgeoise profite désormais du luxe autrefois réservé aux aristocrates (Christ1981). On invente et fabrique autant de sièges.le style est la : néo-renaissance ou le néo-Louis XIV. Une « école de fleurs » s'ouvre aux Beaux-Arts de Lyon destinée à donner des sources d'inspiration aux soyeux.

\subsubsection{Les sièges:}

Caractéristiques des sièges et bois :Aussi à chaque forme de dossier sera adapté un canapé, parfois une chauffeuse, une chaise longue ou une duchesse. Les confidents et les indiscrets sont des créations du second empire. Les copies des styles précédents font florès et les experts d'aujourd'hui peuvent encore être confondus. Les fauteuils sont confortables et profonds ; les supports d'accotoires reculent a nouveau pour acceuillir les robes à crinolines comme sous la Régence, pour faire place aux robes à paniers(feray 1997).Le goût pour les bois sombres fait rechercher l'ébène au grain serré, Le noyer, l'acajou veiné, l'amarante et le poirier noirci.

\subsubsection{Les tissus:}

- Genres des tissus: Damas, lampas, dans la Tapisserie on sent une influence Anglaise (le siècle victorien). II s'apparente au style néo-classique anglais en plus orné. Velours et soie. Le capiton connut son apogée . Exécutés dans tous les tissus : velours, percale, satin ou soie, imprimé ou uni, passementeries, glands, cartisanes, câblés. Toile de Jouy de toutes les époques, cotonnades imprimées, le satin fermière (tissus a fond noir ou grenat), la percale glacée Fig11.

- Ornements des tissus: motifs de styles Louis XV et Louis XVI, trés charges, grosses fleurs, chinoiseries, capitons, pois.La période est égalementcaractérisée par des tissus à f leurs, les motifs de fleurs en bouquet rond, à panier, en guirlande sont les principaux éléments décoratifs de ces tissus. Toutefois les colonies apportent leur part en motifs orientaux (Asie surtout), floraux sur fond noir. Beaucoup d'uni mais également des imprimés variés : fleurs, grands ramages, verdures exotiques (Ossut 2002).

- Couleurs des tissus : Généralement clairs (jaunes, crème, écrus, verts pâles) ils sont unis, ornés de grands ramages multicolores ou de larges compositions florales (fleurs des champs, roses et pivoines).foncées bordeaux, bleu foncé, vert foncé, brun (en accord avec le bois), tous les rouges, les contastes avec le noir.

Fig. 11 Fauteuil Napoleon III

Source: Ossut,C. (2002)p.85

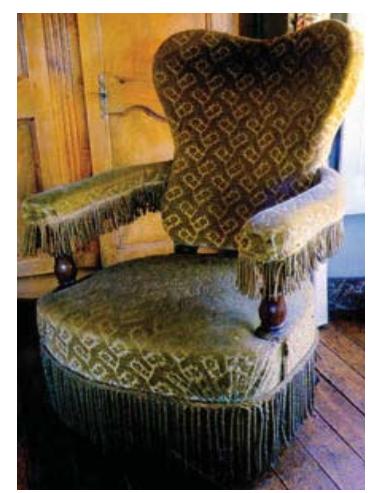

5.10 Le style Art-Nouveau (Modern Style) 1900-1919

5.10.1 La dimension culturelle influant les sièges:

Au XXème siècle, un courant novateur puissant, formant d'étranges entrelacs parmi de nombreuses sources 
d'inspiration : le style nélo-gothique, les peintres préraphaélites, les estampes japonaises, les verriers, les écrivains voyageurs amoureux de Byzance et de l'Orient. II prend comme modèle la nature dont il veut capter la vie en s'inspirant de ses lignes mouvantes. II se caractérise par la ligne asymétrique.La bourgeoisie est la seule à s'intéresser à la création (Loze 2000). . Le Modern Style ou Art Nouveau s'inspire du monde végétal. II fit revivre l'artisanat et éloigne celui de la machine

\subsubsection{Les sièges:}

Caractéristiques des sièges et bois :Les chaises s'inspirent des lignes courbes, galbées et évasées Fig 12. II utilise les formes sinueuses des lianes, du liseron ou des nénuphars. Ces mouvements ondulants se réfèrent aussi à la souplesse de la chevelure féminine. L'art nouveau est réalisé la plupart du temps dans des bois exotiques comme le tamarinier, l'acajou du Brésil ou le citronnier( Christ1981).

\subsubsection{Les tissus:}

- Genres des tissus: les étoffes deviennent moins précieuses qu'au début du siècle. On préfère le lampas, Le damas, les étoffes brochées et la brocatelle sont encore d'usage mais cohabitent avec les velours (de laine ou coton) et des étoffes imprimés sur toile ou satin.L'Art Nouveau revient pour les modèles de luxe aux riches soieries.

- Ornements des tissus: Les thèmes principaux du Moderne Style sont d'inspiration végétale. Les motifs utilisés sont reliés à la nature, les fleurs (nénuphar, iris, orchidées...), les insectes (papillon, libellules...), des oiseaux, des hippocampes, des serpents, des lézards...et se retrouvent sur les ouvrage d'ébénisterie, de verrerie, de tissus et d'imprimés divers, de ferronnerie d'art .Plantes et corps de femme et la chevelure féminine, sont sculptés sur les meubles qui ne tolèrent les surfaces planes qu'ornées de marqueterie, paysage, semblables à des tableaux, avec l'omniprésence de la flore et la faune symbolique. D'un coté ces lignes sinueuses sont isolées et dessinées comme en filigrane, laissant de grandes parties du fond visibles. L'autre traitement à l'opposé bien qu'utilisant aussi ces mêmes formes sinueuses remplira toute la surface de la tapisserie en un entremêlement inextricable (Morand 1973).

- Couleurs des tissus : Les couleurs dominantes sont les bleu, vert, gris, rose, mauve, beige, au ton doux et pastel. ... Les couleurs seules jouant le rôle de contraste et facilitant ainsi la lecture du motif.

Fig. 12 Siège Art Nouveau (Hector Guimard)

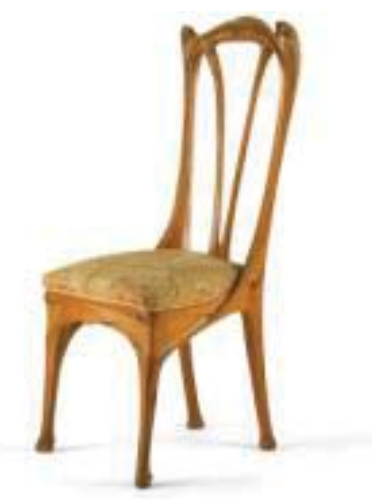

Source: http://www.confort-decor.ch(acces 26/5/2013

5.11 Le style Art-Déco (1919-1939)

\subsubsection{La dimension culturelle influant les sièges:}

Avec le style Art déco, s'achève l'histoire des styles, qui donne la primauté à la ligne droite sur les courbes et les 
contre-courbes ou les créateurs s'orientent vers des lignes pures et sobres. Tois périodes se distinguent dans le style Art Déco: Une époque de renouveau (1910-1923), une autre que l'on peut nomer "Floral" (1919-1931), une dernière enfin caractérisée par le modernisme et le géométrique (1923-1936).Les ébénistes s'inspirent les uns du Louis XV ou Louis $\mathrm{XVI}$, les autres de la Restauration, d'autres tentent d'opérer une synthèse des styles classiques, ou vont chercher leurs references en Orient ou en Espagne.La decouverte de l'Afrique noire, de la civilization américaine, des voyages rapides et du soleil de la Côte d'Azur a eu des influences sur l'inspiration ornemntale des stylistes et des ébénistes, donc un style hétéroclite avec un vif souci d'imagination.

\subsubsection{Les sièges:}

Caractéristiques des sièges et bois :On assiste a une prolifération de formes sans liens entre elles et puisant a toutes les sources historiques.Une etude rationnelle du confort . les pieds fuselés, galbés ou souvent fortement inclinés, sont minces. Les chaises d'inspiration rustique sont nombreuses.Le cannage est employé . le fauteuil club, inspiré de modèles anglais est à la mode ; entièrement recouvert de cuir ou de fourrure( Melagati2010). le bois peint ou plaqué, ciré ou naturel; les plus employés sont l'acajou sombre, le palissandre,L'ebene de Macassar aux zébrures clairs, ainsi que des bois moins précieux, chene,noyer, merisier,Des filets d'ivoire, des incrustationsde metal, des laques de couleur Fig 13.

\subsubsection{Les tissus:}

- Genres des tissus: Autour de 1930 l'industrialisation de la production du mobilier ne permet pas à la tapisserie de siège de s'adapter à toute la profusion créative. Néanmoins pour le mobiler confortable des paquebots, celui des hotels de luxe et bien sûr celui des hotels particuliers oû maisons bourgeoises, de grands artistes dont Raoul Duffy créeront des dessins pour les textiles et y laisseront de superbe composition. L'art nègre est à la mode et on retrouvera beaucoup de cette inspiration dans la décoration des tissus et de la tapisserie de siège. L'Art Déco revient pour les modèles de luxe aux riches soieries. velours - velours froissé - voilages- le cuir.

- Ornements des tissus :La femme et la nature restent des sources d'inspiration mais leurs contours sont stylisés, a la limite d'un symbolisme qui tend vers l'abstraction.La "rose-Iribe", en 1908, un motif de rose stylisée qui restera l'un des symboles de la période "Art Déco", la rose, aux pétales géometriques enserrés dans des feuilles en triangle( Ossut 2002).

Fig. 13 Siège style Art Déco (Clément Rousseau) Paris, vers 1921 Palissandre, galuchat teinté vert, ivoire, garniture en soie gris bleu clair

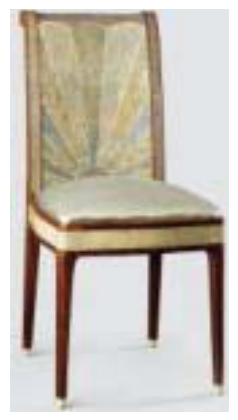

Source: http://www.lesartsdecoratifs.fr/francais/arts-decoratifs/collections

Durant cette période ,on utilise moins la tapisserie de siège. De grands artistes ; Raoul Duffy s'intéressent aux textiles d'ameublement et font de superbes compositions. L'art nègre est à la mode qu' on retrouvera dans la décoration des tissus. D'autres motifs sont très en vogue tels ces décors floraux en demi teintes et sur fond sombre. Ces décorations des tapisseries de siège sont toujours d'un graphisme et d'une composition très stylisés. 
- Couleurs des tissus : profondes ou demi-teinte (noir, blanc, rose, champagne, abricot...)

Fig. 14 Damas de soie style Art Déco

Fig. 15 Soie Artificielle 1925style Art Déco
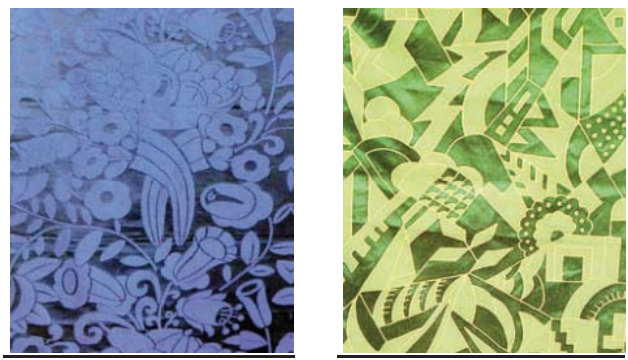

Source: Ossut,C. (2002)p.94

\section{Résultats de la recherche}

1. La dimension culturelle de la société representée par les pensées artistiques, politiques, économiques et même sociale à travers les époques subit une influence majeure sur la philosophie du design et par conséquent sur la relation entre le design du textile (ornements, motifs, couleurs, texture) et le style du mobilier.

2. Le designer ou l'architecte d'intérieur doit avoir une bonne reconnaissance du mobilier de style et des textiles associés, cela contribut à la bonne création de l'atmosphère des différents styles dans l'espace.

3. Le siège perd une grande partie de sa valeur esthétique en cas de la méconnaissance du tissu qui s'adapte bien à la forme et au style du siège .

\section{Recommandations}

1. L'architecte d'intérieur doit de plus en plus développer des qualités d'ensemblier entre l'univers du siège et l'univers du décor pour apporter confort, bien-être et harmonie au cadre de vie dans l'habitat.

2. II faut encourager les designers du textile à reproduire la philosohie des ornements des styles au moyen des matériaux qui allient entre la bonne qualité ,le meilleur prix à la lumière et la technologie moderne.

\section{Références}

Alcouffe,D.Lefebrure,A.(1998).Le Mobilier du Musee du Louvre.Tome(2). Paris: Faton

Bedel, J.(2004).Le Grand Guide des styles.Paris. Hachette.

Castellucio, S. (2002). Le style Louis XIII. Paris : Amateur.

Christ, Yvan. (1981). « La grammaire des styles, L'Art au XIXe siècle, du Second Empire à la fin du siècle ». Paris: Flammarion.

Demetrescu ,C.(2002). Le Style Louis XIV.Paris: Editions de l'Amateur.

Demetrescu ,C. (2003). Le Style Régence .Paris: Editions de l'Amateur.

Droguet , A(2004). Les Styles Transition et Louis XVI .Paris: Editions de l'Amateur.

Feray, J.(1997). Architecture intérieure et décoration en France, des origines à 1875.Paris: Berger-Levrault.

http://www.lesartsdecoratifs.fr/francais/arts-decoratifs/collections-26/parcours-27/chronologique/art-nouveau-art-deco/les-salles-300/lesfondements-de-I-art-deco/chaise-854

http://www.confort-decor.ch/29616/29643.html

http:/www./tldtouteladecoration.com/2012/03/11/etoffes-du-xviieme-louis-xiv/

http://www.maison-salamandre.com

http://www.prelle.fr/files/pdf/presse/2013

http://www.cdma.greta.fr/

http://www.tapisseriedefrance.fr

http://www.lesartsdecoratifs.fr 
http://www.moinat.net/a-propos

http://www.louvre.fr/oeuvre-notices/paire-de-fauteuils-la-reine-

Jarry .M , Devinoy. P.( 1973). Le siège français. Paris:Office Du Livre.

Kjellberg, P.(1991).Le Meuble francais et européen du Moyen Âge à nos jours.Paris: Editions de l'Amateur.

Loze, Pierre. (2000). L'Art nouveau. France: Flammarion.

Melagati, L.(2010). Comment identifier le mobilier. De la Renaissance aux Arts-déco. Paris:Hazan.

Morand, A.(1973). L'encyclopédie Des Styles D'hier Et D'aujourd'hui .Du Louis XIII Au Restauration. Paris: Marabout.

Mouquin,S(2003). Le Style Louis XV.Paris: Editions de l'Amateur.

Ossut,C. (2002).Le siège et sa garniture.Paris :Vial.

Ossut,C. (1983). Tapisserie d'ameublement.Paris :Vial.

Quette,A.M.(1996). Mobilier Louis XIII, Louis XIV. Paris:Massin.

Wiuegandt ,C.P .(1994).Régence Louis XV. Paris:Massin. 